\title{
Unsteady contact melting of a rectangular cross-section material on a flat plate
}

\author{
T. G. Myers, ${ }^{1, a)}$ S. L. Mitchell, ${ }^{2}$ and G. Muchatibaya ${ }^{1}$ \\ ${ }^{1}$ Department of Mathematics and Applied Mathematics, University of Cape Town, \\ Rondebosch 7701, South Africa \\ ${ }^{2}$ Department of Mathematics and Statistics, MACSI, University of Limerick, Limerick, Ireland
}

(Received 15 February 2008; accepted 4 September 2008; published online 6 October 2008)

\begin{abstract}
The work in this paper concerns a mathematical model of the contact melting process of a rectangular material in contact with a hot plate. The problem is described by a coupled system of heat equations in the solid and melt layer, fluid flow in the melt, a Stefan condition at the melt interface, and a force balance between the weight of the solid and the fluid pressure. Since the melt layer remains thin throughout the process, we use the lubrication approximation to the fluid equations and assume that the heat flow in the fluid is dominated by conduction across the thin film. In the solid we employ a heat balance integral method. Results show that the film height has initial and final rapid increases, whereas for intermediate times the height slowly increases. The quasisteady state of previous models is never attained: This is shown to be an effect of neglecting the change in mass and conduction in the solid. The previously observed initial infinite velocity of the melt is shown to be a result of the perfect thermal contact assumption. For a water-ice system the melting rate is shown to be approximately linear, this allows us to reduce the problem to solving a single first order differential equation for the liquid layer thickness. The main analysis is carried out in two dimensions, but we briefly highlight the extension to three dimensions. The method is verified by comparison with previously published experimental results on the melting of n-octadecane. (C) 2008 American Institute of Physics. [DOI: 10.1063/1.2990751]
\end{abstract}

\section{INTRODUCTION}

Contact melting is the process whereby a phase change material (PCM) is placed in contact with a surface that is at a temperature above the phase change temperature. This leads to melting of the PCM, so a fluid layer forms between the two surfaces. The weight of the free solid acts to squeeze out the liquid and so the melt layer remains thin. Since the melting is driven by heat transfer through the fluid layer the fact that it remains thin results in a high heat flux and a melting rate that is several times higher than buoyancy induced melting, see Refs. 1 and 2, for example (Moallemi et $a .^{3}$ quoted a figure of seven to eight times higher for their experiments on $n$-octadecane).

Contact melting occurs in situations such as process metallurgy, geology, and nuclear technology. ${ }^{4-6}$ Certain thermal storage systems rely on the storage of energy as latent heat, which is released upon melting. In this case contact melting occurs as the frozen solid melts due to contact with the hot container wall. $^{2,7-9}$ Many examples of contact melting are provided in the review of Bejan. ${ }^{10}$ Perhaps the two most familiar examples of contact melting are the Leidenfrost effect and ice skating. The Leidenfrost effect occurs when a liquid comes into contact with a substrate which is at a temperature significantly above the boiling point. An insulating vapor layer forms which allows the liquid to float above the solid and results in much slower evaporation of the drop than

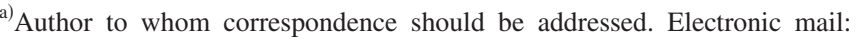
tim.myers@ymail.com.
}

if it remained in contact with the surface. ${ }^{11,12}$ Much of the classical work in the field of contact melting was carried out by Bejan in relation to sliding on ice and snow, see Refs. 10 and 13 , for example.

In the past, mathematical models of contact melting have generally relied on the following assumptions:

(1) The temperature of the solid remains at the melting temperature $T_{m}$ throughout the process.

(2) The melting process is in a quasisteady state, that is, if the position of the melt front is denoted $h(t)$ then $h_{t}=0$.

(3) Heat transfer in the liquid is dominated by conduction across the film.

(4) The lubrication approximation holds in the liquid layer, so the flow is primarily parallel to the solid surface and driven by the pressure gradient. The pressure variation across the film is negligible.

(5) The amount of melted fluid is small compared to that of the initial solid.

(6) There is a perfect thermal contact between the liquid and substrate or there is a constant heat flux.

See Refs. 1, 2, and 10.

Of course there exist papers where certain of these restrictions are relaxed. Litsek and Bejan ${ }^{14}$ attempted to remove assumption (1) by incorporating a convection term in the solid heat equation. Their expression for the temperature gradient at the melting front of the solid is then constant. This result is far from realistic. If the solid is assumed to be at a constant temperature $\theta_{0}$ which is below the melt tem- 
perature, $\theta_{0}<T_{m}$, then the resulting expression for the melting rate differs from results where $\theta_{0}=T_{m}$ with the change $L_{m} \rightarrow L_{m}+c_{s}\left(T_{m}-\theta_{0}\right)$, where $L_{m}$ is the latent heat of melting and $c_{s}$ is the heat capacity of the solid, see Refs. 1 and 3, for example. Assumption (2) is removed by Yoo $^{15}$ who showed that in the case of perfect thermal contact the initial velocity of the block is infinite. For both perfect thermal contact and constant flux the melt thickness initially increases rapidly before reaching a constant height. Groulx and Lacroix ${ }^{1}$ also attempted to remove assumption (3). They include the vertical convection term in the liquid. The vertical velocity $w(z)$ required for this may be obtained through the lubrication analysis, however, to permit an analytical solution they take $w$ to be a constant equal to the melting rate. Since $w=0$ at the substrate $z=0$, except at the melting interface, their choice of $w$ is too high. Perhaps a better constant approximation would be the average value of $w$ across the film. Yoo et al. ${ }^{16}$ retained the full expression for $w(z)$ and so obtained a result in terms of an exponential integral, which must be evaluated numerically. Moallemi $e t a l^{3}$ used an integrated form of the heat equation including vertical convection and then impose a quadratic temperature in order to make progress. This process is, in fact, an application of the heat balance integral (HBI) method of Goodman ${ }^{17}$ that is used in later sections of this paper to determine the temperature in the solid. All researchers agree that assumption (4), namely, the lubrication approximation, is appropriate. Moallemi et l. $^{3}$ have a decreasing mass model; this is discussed further in Sec. V B. Hirata and Makino ${ }^{18}$ used an iterative technique to calculate the mass at each time step. All researchers assume perfect thermal contact or constant heat flux.

Lacroix ${ }^{19}$ recognized the shortcomings of current models in a study of the melting of a parallelepedic block with a varying mass and temperature within the solid [so removing assumptions (1), (2), and (5)]. Unfortunately the paper is unclear on a number of points. The force balance indicates that the PCM is floating in the melt, although there is no channel allowing the melt to move around the PCM. The linear temperature profile assumed in the bottom and top liquid layers requires a thin film approximation. This will hold in the lower layer provided the fluid removal is sufficiently rapid. In the top layer it is unlikely, particularly when the top layer is thicker than the solid. There appears to be some confusion between the melting rate and the velocity of the melt interface. Due to the squeezing effect the velocity of the interface differs from the melting rate yet these quantities appear to be identical, see their Eqs. (4) and (15). Finally, the heat equation in the solid is solved using separation of variables. However, the solid boundaries are time dependent so the "constant" of separation is also a function of time and the equation is not separable. Hence we will not use results from this paper for comparison purposes, although, since it is the paper where most of the standard restrictions are removed, in later sections we will point out qualitative similarities.

In reality the temperature in the solid is unlikely to be at the melt temperature and rather than being constant it will decrease away from the melt front. Yoo ${ }^{15}$ has already noted that initially the melt height is far from constant. For sufficiently small times the melted mass must be much less than

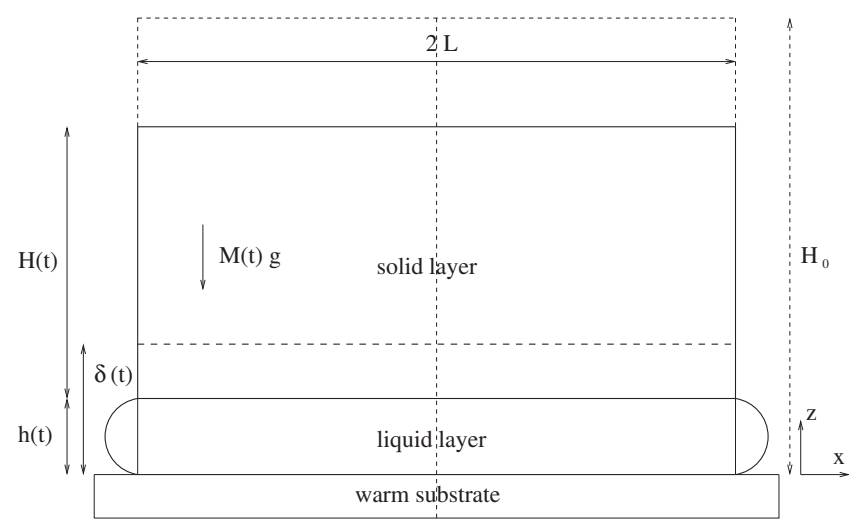

FIG. 1. Schematic diagram for contact melting.

the mass of the solid, but as the melting progresses this will change and at some stage the melted mass will be greater than that of the solid. Consequently, the solid mass $M(t)$ must be a decreasing function of time (and this has an effect on the quasisteady height). Finally, perfect thermal contact with the substrate is an unlikely scenario; Newton cooling is more realistic. In light of these points, in the following work we will study contact melting without invoking assumptions (1), (2), (5), and (6).

Although in recent studies it is standard to investigate the contact melting of a sliding three-dimensional block we will focus on the base problem, namely, the stationary twodimensional situation. The appropriate extension to three dimensions is simple, leading to a standard Couette term in the lubrication approximation for the horizontal velocity and the introduction of a function of the base area found by solving Poisson's equation for the pressure. The extension to three dimensions will be briefly discussed in Sec. VI. In the following section we will describe the governing equations and the approximations that are made in deriving them. We will retrieve quasisteady solutions from these equations for comparison with the subsequent unsteady solutions. In Sec. IV we discuss the solution to the thermal problem, which is tackled via the HBI method, see Refs. 17 and 20. Finally, we show results for the melting of ice on a warm surface and discuss the differences between the present and previous models. Experimental confirmation of the method is achieved by comparing results with data from the melting of $n$-octadecane published by Moallemi et al. ${ }^{3}$

\section{PROBLEM DESCRIPTION}

In this section we describe the governing equations for a finite thickness block of PCM placed on a substrate that is above the phase change temperature, as depicted in Fig. 1. Energy is supplied by the substrate and this causes the block to melt. The block has length $2 L$ and height $H(t)$. If $h_{m}(t)$ denotes the thickness of melted solid then the height is $H(t)=H_{0}-h_{m}(t)$, where $H_{0}=H(0)$ is the initial block thickness. The mass per unit width is $M(t)=2 \rho_{s} L\left[H_{0}-h_{m}(t)\right]$. The melt layer occupies the region $z \in[0, h(t)]$. The height of the melt layer $h(t)$ differs from $h_{m}$ since fluid is squeezed out at the edges $x= \pm L$. The pressure at either side of the block is ambient; without loss of generality we can set this to zero, 

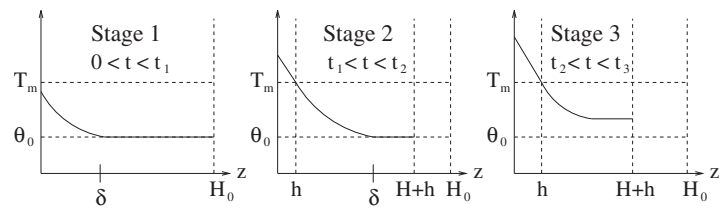

FIG. 2. Schematic of the three stages of melting when a block is placed on a surface above the melting temperature.

$p( \pm L)=0$. The solid and liquid layer temperatures are denoted $\theta$ and $T$, respectively. Initially, the block is at a constant temperature $\theta_{0}$, which is below the melting temperature, $\theta_{0}<T_{m}$. Except in the limit of perfect thermal contact the melting process occurs in three distinct stages, as depicted in Fig. 2. Initially the bottom of the block heats up until the region in contact with the substrate reaches the melting temperature. We denote this as stage 1. Melting only begins at the end of stage 1. Stage 2 defines the time when there is a region inside the block still close to the initial temperature $\theta_{0}$. Finally, stage 3 occurs when the temperature inside the block is everywhere significantly above the initial temperature. Clearly there are situations where the top of the block also starts to melt. This could be incorporated by following the analysis of Myers et al. ${ }^{20}$ however, to avoid confusion and to isolate the effect of heating from the substrate, in the present study we restrict our attention to the simpler problem. To be clear we define stage 1 as occurring from $t$ $\in\left[0, t_{1}\right]$, where $t_{1}$ comes from solving $\theta\left(0, t_{1}\right)=T_{m}$. Stage 2 lasts for $t \in\left[t_{1}, t_{2}\right]$ and $t_{2}$ defines when the temperature $\theta(H$ $\left.+h, t_{2}\right)$ first rises above $\theta_{0}$. Since the heat equation has infinite speed of propagation theoretically this occurs at $t=0$. However, the HBI method defines a heat penetration depth $\delta(t)$, which measures where the temperature rise becomes negligible (this is discussed in detail later). The time $t_{2}$ occurs when the heat has penetrated all the way through the block, $\delta\left(t_{2}\right)=H\left(t_{2}\right)+h\left(t_{2}\right)$. Stage 3 continues from $t_{2}$ until the block has completely melted, $t \in\left[t_{2}, t_{m}\right]$.

\section{A. Governing equations}

The mathematical model requires the determination of the flow in the liquid layer. The amount of liquid depends on the melting rate, which involves the Stefan condition, and this involves the temperature gradient in both the solid and liquid. Consequently we must solve the following system:

$$
\begin{aligned}
& \eta \frac{\partial^{2} u}{\partial z^{2}}=\frac{\partial p}{\partial x}, \quad \frac{\partial p}{\partial z}=0, \quad \frac{\partial u}{\partial x}+\frac{\partial w}{\partial z}=0, \\
& \frac{\partial^{2} T}{\partial z^{2}}=0, \\
& \frac{\partial \theta}{\partial t}=\kappa_{s} \frac{\partial^{2} \theta}{\partial z^{2}}, \\
& \rho_{s} L_{m} \frac{d h_{m}}{d t}=\left.k_{s} \frac{\partial \theta}{\partial z}\right|_{z=h}-\left.k_{l} \frac{\partial T}{\partial z}\right|_{z=h},
\end{aligned}
$$

$$
2 \rho_{s} L\left[H_{0}-h_{m}(t)\right] g=\int_{-L}^{L} p d x .
$$

Equations (1) represent the standard lubrication approximation for the fluid flow, where $\eta$ is the dynamic viscosity and $(u, w)$ is the velocity vector. Where it is necessary to distinguish between the phases we use subscript $l$ to denote liquid and $s$ for solid. For realistic situations the largest term neglected in this approximation is $\mathcal{O}\left(\epsilon^{2} \mathrm{Re}\right)$, where, if $U$ is the velocity scale and $\mathcal{H}_{l}$ the height scale, the Reynolds number $\mathrm{Re}=\rho_{l} U L / \eta$ and $\epsilon=\mathcal{H}_{l} / L$. Equations (2a) and (2b) are the heat equations in liquid and solid, respectively. Since the liquid layer remains thin we assume that heat transfer is conduction dominated. The largest term neglected is $\mathcal{O}\left(\epsilon^{2} \mathrm{Pe}\right)$, where the Peclet number $\mathrm{Pe}=U L / \kappa_{l}$, where $\kappa$ is the thermal diffusivity. This is consistent with the approximations of Bejan. ${ }^{10}$ In the solid we neglect diffusion in the $x$ direction for two reasons. First, in general, the scaling shows it to be small and second (and most significantly) there is no mechanism for temperature variation in the $x$-direction in the solid. This is due to the fact that the sides and top of the block are insulated and, before melting occurs, the temperature at $z$ $=0$ depends on the substrate temperature, which is independent of $x$. Once melting starts $\theta=T_{m}$ is constant at $z=h$. Equation (3) is the Stefan condition, where $k$ is the thermal conductivity, and Eq. (4) is a force balance where we have neglected the acceleration term, which requires assuming that $\mathcal{H}_{l} /\left(g \tau^{2}\right)$ is small, where $\tau$ is the time scale. The left hand side represents the weight of the solid and the right hand side is the fluid pressure acting to support this weight. In Sec. $\mathrm{V}$ we will verify that all the neglected terms are indeed small.

The flow equations require solving subject to

$$
\begin{aligned}
& \left.u\right|_{z=0}=\left.w\right|_{z=0}=0,\left.\quad u\right|_{z=h}=0, \\
& \left.w\right|_{z=h}=\left(1-\frac{\rho_{s}}{\rho_{l}}\right) \frac{d h}{d t}+\frac{\rho_{s}}{\rho_{l}} W(t),
\end{aligned}
$$

where $W(t)=d h / d t-d h_{m} / d t$ is the vertical velocity of the solid, defined as the difference between the liquid layer growth rate and the melt rate. Hence

$$
\left.w\right|_{z=h}=\frac{d h}{d t}-\frac{\rho_{s}}{\rho_{l}} \frac{d h_{m}}{d t} .
$$

These conditions represent no slip at $z=0$. At $z=h$ there is again no slip in the $x$ direction, a mass balance in the $z$ direction leads to the final condition (more details of this form of mass balance are given by Myers et al. $^{21,22}$ ). In the absence of melting $w=d h / d t$ is the standard squeeze velocity. An equivalent expression to Eq. (5) is given in Ref. 16. The pressure is ambient at either end of the block,

$$
p(-L)=p(L)=0 \text {. }
$$

The thermal problem is subject to different conditions in the different stages. Initially, during stage 1, no melting occurs and so a cooling condition is applied to the solid, 


$$
\frac{\partial \theta}{\partial z}=\frac{-q+h_{s s}\left(\theta-T_{s}\right)}{k_{s}} \quad \text { at } z=0,
$$

where $q$ represents a heat flux from the substrate, $T_{s}$ is the substrate temperature, and $h_{s s}$ is the heat transfer coefficient between the solid and substrate. Immediate melting only occurs if $h_{s s} \rightarrow \infty$. At $z=H(t)+h(t)$ the surface is insulated and so we apply

$$
\left.\frac{\partial \theta}{\partial z}\right|_{z=H+h}=0
$$

note in stage $1 H+h=H_{0}$. We do not impose a cooling condition here since this complicates the algebra and consequently obscures the main features of the melting process that we wish to highlight. The extension to a cooling condition at $z=H+h$ can easily be calculated following the work described in Ref. 20. When water appears (on the surface $z$ $=0)$ at the end of stage 1 a cooling condition is applied to the liquid,

$$
\frac{\partial T}{\partial z}=\frac{-q+h_{s l}\left(T-T_{s}\right)}{k_{l}} \quad \text { at } z=0,
$$

where $h_{s l}$ is the heat transfer coefficient between the liquid and substrate. In the limit $h_{s l} \rightarrow \infty$ we retrieve the standard condition $T=T_{s}$ at $z=0$. When $h_{s l}=0$ we retrieve the constant flux condition. The melting interface remains at the melting temperature $T_{m}$, and so

$$
\theta=T=T_{m} \quad \text { at } \quad z=h .
$$

Since the flow equations are only coupled to the thermal problem through the definition of their domain $z \in[0, h(t)]$, we can immediately solve for the velocities and the pressure. The velocities in the $x$ and $z$ directions are

$$
u=\frac{p_{x}}{2 \eta} z(z-h), \quad w=-\frac{p_{x x}}{12 \eta} z^{2}(2 z-3 h) .
$$

The boundary condition on $w(h, t)$, Eq. (6), leads to an expression for $p_{x x}$ which can be solved using boundary conditions (7) to give

$$
p=\frac{6 \eta}{h^{3}}\left(\frac{d h}{d t}-\frac{\rho_{s}}{\rho_{l}} \frac{d h_{m}}{d t}\right)\left(x^{2}-L^{2}\right) .
$$

Substituting Eq. (13) into the force balance (4) leads to

$$
2 \rho_{s} L\left[H_{0}-h_{m}\right] g=-\frac{8 \eta L^{3}}{h^{3}}\left(\frac{d h}{d t}-\frac{\rho_{s}}{\rho_{l}} \frac{d h_{m}}{d t}\right) .
$$

\section{STANDARD QUASISTEADY ANALYSIS}

Before proceeding to the full problem we first consider the standard quasisteady solution but use the general cooling condition (6). The standard quasisteady analysis requires an isothermal solid, a fixed melt layer thickness, $h_{t}=0$, and a fixed solid mass. Assumptions (1), (2), and (5) reduce the Stefan condition and force balance, Eqs. (3) and (14), to

$$
\begin{aligned}
& \rho_{s} L_{m} \frac{d h_{m}}{d t}=-\left.k_{l} \frac{\partial T}{\partial z}\right|_{z=h}, \\
& 2 \rho_{s} L H_{0} g=\frac{8 \eta L^{3}}{h^{3}} \frac{\rho_{s}}{\rho_{l}} \frac{d h_{m}}{d t} .
\end{aligned}
$$

Since $\theta=T_{m}$, any input of energy causes melting, so there is no stage 1 for this problem.

Integrating Eq. (15b) gives

$$
h_{m}=\frac{H_{0} \rho_{l} g h_{q}^{3}}{4 \eta L^{2}} t \text {, }
$$

where $h_{q}$ denotes the quasisteady melt height. Without the squeeze effect $h_{m} \sim \sqrt{t}$, see Ref. 23, for example, so it is clear that the squeeze effect leads to more rapid melting. To determine $h_{q}$ we must look at the thermal problem. The temperature in the liquid is

$$
T=T_{m}-\frac{q+h_{s l}\left(T_{s}-T_{m}\right)}{k_{l}+h_{s l} h}(z-h),
$$

which is found by solving Eq. (3) with boundary conditions (10) and (11). Substituting into the Stefan condition (15a) gives

$$
\rho_{s} L_{m} \frac{H_{0} \rho_{l} g h_{q}^{3}}{4 \eta L^{2}}=k_{l} \cdot \frac{q+h_{s l}\left(T_{s}-T_{m}\right)}{\left(k_{l}+h_{s l} h_{q}\right)} .
$$

This provides a quartic equation for the quasisteady height $h_{q}$, which is easily solved numerically. The melt height then follows from Eq. (16). In the limit $h_{s l} h_{q} \ll k_{l}$ we obtain

$$
h_{q} \approx \sqrt[3]{\frac{4 \eta L^{2}\left[q+h_{s l}\left(T_{s}-T_{m}\right)\right]}{\rho_{s} \rho_{l} L_{m} H_{0} g}} .
$$

Setting $h_{s l}=0$ in Eq. (19) we retrieve a form of the constant flux solution of Ref. 15. In the limit of perfect thermal contact, $h_{s l} \rightarrow \infty$, we retrieve the standard quasisteady result for an isothermal block, subject to $T(0, t)=T_{s}$,

$$
h_{q \infty}=\sqrt[4]{\frac{4 \eta L^{2} k_{l}\left(T_{s}-T_{m}\right)}{\rho_{s} \rho_{l} L_{m} H_{0} g}} .
$$

Note that there is a qualitative difference between these two expressions. If we assume perfect thermal contact then $h$ $\sim\left(T_{s}-T_{m}\right)^{1 / 4}$. The more realistic condition of finite $h_{s l}$ gives $h \sim\left(T_{s}-T_{m}\right)^{1 / 3}$.

In Table I we present appropriate parameter values for the melting of an ice block on a substrate at $298 \mathrm{~K}$, see Refs. 24-26, for example. The heat transfer coefficients $h_{s s}=763$ and $h_{s l}=855 \mathrm{~W} / \mathrm{m}^{2}$ were obtained through simple experiments, which are described in detail in Ref. 20. For a block with $H_{0}=0.05 \mathrm{~m}, L=0.1 \mathrm{~m}$, and $\Delta T=25$ we find from Eq. (18) $h_{q} \approx 1.98 \times 10^{-4} \mathrm{~m}$, whereas for perfect thermal contact $h_{q \infty} \approx 2.48 \times 10^{-4} \mathrm{~m}$. The second set of data in Table I are for $n$-octadecane, which is discussed in Sec. V B. The values are taken from Refs. 2 and 3.

For the melting of a block at a constant temperature below the melting temperature, $\theta_{0}<T_{m}$, it is standard to replace $L_{m}$ with $L_{m}+c_{s}\left(T_{m}-\theta_{0}\right)$ in the expression for $h_{q^{\infty}}$, see 
TABLE I. Parameter values for ice/water and liquid/solid $n$-octadecane.

\begin{tabular}{|c|c|c|c|c|c|}
\hline \multicolumn{6}{|c|}{ Ice/water } \\
\hline$k_{l}$ & 0.57 & $\mathrm{~W} \mathrm{m^{-1 }} \mathrm{K}^{-1}$ & $k_{s}$ & 2.18 & $\mathrm{~W} \mathrm{~m}{ }^{-1} \mathrm{~K}^{-1}$ \\
\hline$\rho_{l}$ & 1000 & $\mathrm{~kg} \mathrm{~m}^{-3}$ & $T_{s}$ & 298 & K \\
\hline$\rho_{s}$ & 917 & $\mathrm{~kg} \mathrm{~m}^{-3}$ & $\eta$ & 0.001 & $\mathrm{~N} \mathrm{~s} \mathrm{~m}^{-2}$ \\
\hline$L_{m}$ & $3.34 \times 10^{5}$ & $\mathrm{~J} \mathrm{~kg}^{-1}$ & $\kappa_{l}$ & $1.35 \times 10^{-7}$ & $\mathrm{~m}^{2} \mathrm{~s}^{-1}$ \\
\hline$T_{m}$ & 273 & K & $\kappa_{s}$ & $1.16 \times 10^{-6}$ & $\mathrm{~m}^{2} \mathrm{~s}^{-1}$ \\
\hline$h_{s l}$ & 855 & $\mathrm{~W} / \mathrm{m}^{2}$ & $h_{s s}$ & 763 & $\mathrm{~W} / \mathrm{m}^{2}$ \\
\hline$\theta_{0}$ & 258 & $\mathrm{~K}$ & & & \\
\hline \multicolumn{6}{|c|}{ Solid/liquid $n$-octadecane } \\
\hline$k_{l}$ & 0.15 & $\mathrm{~W} \mathrm{~m} \mathrm{~m}^{-1} \mathrm{~K}^{-1}$ & $k_{s}$ & 0.38 & $\mathrm{~W} \mathrm{m^{-1 }} \mathrm{K}^{-1}$ \\
\hline$\rho_{l}$ & 771.2 & $\mathrm{~kg} \mathrm{~m}^{-3}$ & $T_{s}$ & 298 & K \\
\hline$\rho_{s}$ & 930 & $\mathrm{~kg} \mathrm{~m}^{-3}$ & $\eta$ & 0.0036 & $\mathrm{~N} \mathrm{~s} \mathrm{~m}^{-2}$ \\
\hline$L_{m}$ & $2.435 \times 10^{5}$ & $\mathrm{~J} \mathrm{~kg}^{-1}$ & $\kappa_{l}$ & $9.0 \times 10^{-8}$ & $\mathrm{~m}^{2} \mathrm{~s}^{-1}$ \\
\hline$T_{m}$ & 301.33 & K & $\kappa_{s}$ & $1.9 \times 10^{-7}$ & $\mathrm{~m}^{2} \mathrm{~s}^{-1}$ \\
\hline$\theta_{0}$ & 298 & K & & & \\
\hline
\end{tabular}

Refs. 1 and 18. For the parameter values of Table I this reduces the height to $h_{q_{\infty}} \approx 2.43 \times 10^{-4} \mathrm{~m}$ (a $2 \%$ difference).

\section{UNSTEADY ANALYSIS}

The crux of this problem lies in the estimation of the temperature profile in the solid. The thinness of the liquid layer ensures that the lubrication approximation is valid and that conduction in the $z$-direction is the primary mode of heat transfer in the liquid, so this region is relatively simple. In the solid we cannot use a thin layer approximation. This is the most likely the reason that it has been neglected in previous studies: Yoo ${ }^{15}$ proposed that initial subcooling of the solid may be neglected since it may not affect the fundamental nature of the melting and also because it is hard to model properly. He suggests it as a possible mechanism for the infinite velocity of the melting front that occurs with the quasisteady model at $t=0$. Our subsequent solutions will show that it is the perfect thermal contact assumption that leads to the infinite initial velocity.

To approximate the temperature in the solid we will utilize Goodman's HBI method. ${ }^{17,20,27-29}$ The HBI method involves approximating the temperature in the solid, usually with a low order polynomial, that is defined over a distance termed the heat penetration depth; in the present solution we say the heat penetrates to $z=\delta$, so the heat penetration depth of Goodman is $\delta-h$ in our notation. Beyond $\delta(t)$ the temperature rise above the initial value is assumed negligible. The coefficients in the approximating function are determined via the boundary conditions. The heat penetration depth satisfies a first order ordinary differential equation (ODE) that is obtained by integrating the heat equation between the melt front (or heated interface) and $\delta$.

\section{A. Stage 1: Initial premelting stage, $0 \leq t \leq t_{1}$}

During this initial phase the PCM is heated at $z=0$, until it reaches the melting temperature. The problem is governed by Eq. (2b) subject to conditions (8) and (9). We can determine an exact solution using Laplace transforms, ${ }^{20,23}$

$$
\begin{aligned}
\theta(z, t)= & \theta_{0}+\frac{q-h_{s s}\left(\theta_{0}-T_{s}\right)}{h_{s s}}\left[\operatorname{erfc}\left(\frac{z}{2 \sqrt{\kappa_{s} t}}\right)\right. \\
& \left.-e^{h_{s s} z / k_{s}+\kappa_{s} t\left(h_{s s} / k_{s}\right)^{2}} \operatorname{erfc}\left(\frac{z}{2 \sqrt{\kappa_{s} t}}+\frac{h_{s s} \sqrt{\kappa_{s} t}}{k_{s}}\right)\right] .
\end{aligned}
$$

Melting begins at time $t_{1}$ when the base reaches the melting temperature $\theta\left(0, t_{1}\right)=T_{m}$. However, once we move on to the melting stage we cannot find an exact solution and so, to be consistent with stages 2 and 3, we will also look for a solution in the premelting stage via the HBI method. For melting problems Myers et al. ${ }^{20}$ showed that a cubic polynomial, neglecting the quadratic term, provides a good approximating function. This is motivated by the small argument expansion of the exact solution.

Following Myers et al. $^{20}$ we take an approximating function of the form

$$
\theta=a_{0}+a_{1}(\delta-z)+a_{3}(\delta-z)^{3}
$$

At $z=0$ we impose the cooling condition (8). The heat penetration depth is defined by $\theta(\delta, t)=\theta_{0}$, and for a smooth transition to the constant temperature beyond $\delta$, we set $\theta_{z}(\delta, t)=0$, see Fig. 2(a). These conditions lead to the temperature profile

$$
\theta=\theta_{0}+\frac{q+h_{s s}\left(T_{s}-\theta_{0}\right)}{\delta^{2}\left(3 k_{s}+h_{s s} \delta\right)}(\delta-z)^{3} .
$$

The second part of the HBI method is to integrate the heat equation $(2 \mathrm{~b})$ over the region where the temperature is above $\theta_{0}$, i.e., from $z=0$ to $\delta$. Using the temperature profile of Eq. (23) in the heat equation and integrating, we obtain the ODE,

$$
\frac{d}{d t}\left[\frac{\delta^{2}}{3 k_{s}+h_{s s} \delta}\right]=\frac{12 \kappa_{s}}{3 k_{s}+h_{s s} \delta}, \quad \delta(0)=0,
$$

which has an implicit solution, 


$$
t=\frac{k_{s}^{2}}{12 \kappa_{s} h_{s s}^{2}}\left[\frac{h_{s s} \delta}{2 k_{s}}\left(\frac{h_{s s} \delta}{k_{s}}+6\right)-9 \ln \left(1+\frac{h_{s s} \delta}{3 k_{s}}\right)\right] .
$$

This stage ends when melting begins at $z=0, \theta\left(0, t_{1}\right)=T_{m}$. Equation (23) gives the appropriate value of $\delta\left(t_{1}\right)=\delta_{1}$,

$$
\delta_{1}=\frac{3 k_{s}\left(T_{m}-\theta_{0}\right)}{q+h_{s s}\left(T_{s}-T_{m}\right)}
$$

and the corresponding time comes from Eq. (25). Note in the limits $h_{s s} \rightarrow \infty$ or $\theta_{0}=T_{m}$, then $\delta_{1}=t_{1}=0$ and melting is immediate, in agreement with previous solutions. For the parameter values of Table I we find $t_{1} \approx 1.6 \mathrm{~s}$ and $\delta \approx 5.1 \mathrm{~mm}$.

\section{B. Stage 2: Initial melting stage, $t_{1} \leq t \leq t_{2}$}

Once melting starts the temperature in the water is

$$
T(z, t)=T_{m}-\frac{q+h_{s l}\left(T_{s}-T_{m}\right)}{k_{l}+h_{s l} h}(z-h) .
$$

This is determined by solving the reduced heat equation (2a) subject to Eqs. (10) and (11). Myers et al. ${ }^{20,24}$ provided a more accurate approximation by including correction terms in the perturbation solution. However, this does not include the squeeze film effect and so allows for thicker films (where the linear approximation deteriorates). In the solid the cubic approximation in the HBI method leads to the temperature,

$$
\theta(z, t)=\theta_{0}+\frac{T_{m}-\theta_{0}}{(\delta-h)^{3}}(\delta-z)^{3},
$$

where $\theta(h)=T_{m}, \theta(\delta)=0$, and $\theta_{z}(\delta)=0$. Integrating the heat equation in the solid for $z \in[h, \delta]$ we find

$$
3 \frac{d h}{d t}+\frac{d \delta}{d t}=\frac{12 \kappa_{s}}{\delta-h} .
$$

The temperature expressions (27) and (29) allow us to calculate the temperature gradients for the Stefan condition,

$$
\rho_{s} L_{m} \frac{d h_{m}}{d t}=\frac{3 k_{s}\left(\theta_{0}-T_{m}\right)}{\delta-h}+\frac{k_{l}\left[q+h_{s l}\left(T_{s}-T_{m}\right)\right]}{k_{l}+h_{s l} h} .
$$

Equations (29) and (30) contain three unknowns, $\delta, h_{m}, h$. The system is closed with the force balance, Eq. (14), and solved subject to the initial conditions $\delta=\delta_{1}, h=h_{m}=0$ at $t$ $=t_{1}$. The stage ends when the heat has penetrated all the way through the block, $\delta=H(t)+h(t)=H_{0}+h(t)-h_{m}(t)$ at time $t_{2}$. We denote the thicknesses at this time as $\delta_{2}, h_{2}, h_{m_{2}}$.

If the PCM is initially at $\theta_{0}=T_{m}$ then the temperature remains constant throughout the solid for all time. The first term on the right hand side of Eq. (30) is identically zero. The second term only becomes infinite at $t=t_{1}=0$ in the limit $h_{s l} \rightarrow \infty$. Consequently from Eq. (14) we see that the infinite velocity $h_{t}$ observed in previous studies is a result of the perfect thermal contact assumption, not the subcooling.

\section{Stage 3: Final melting stage, $t_{2} \leq t \leq t_{3}$}

Once the heat penetration depth reaches the top of the block we can no longer impose $\theta=\theta_{0}$ at $z=\delta$. Instead we impose $\theta_{z}=0$ at $z=H+h$ and find a temperature profile,

$$
\theta=a_{0}-\left(T_{m}-a_{0}\right) \frac{(z-H-h)^{3}}{H^{3}},
$$

where $a_{0}(t)$ replaces $\delta$ as our third unknown. We may now proceed in two ways. We can substitute $\theta$ into the heat equation and integrate as in the previous section, to obtain an ODE for $a_{0}$. This may be solved subject to $a_{0}\left(t_{2}\right)=\theta_{0}$ [which follows by continuity of temperature at $t=t_{2}, \theta\left(H+h, t_{2}\right)$ $=\theta_{0}$ ]. However, we choose to follow Goodman and introduce a function $\phi$, where

$$
\phi=\int_{h}^{H+h} \theta d z=\frac{H}{4}\left(T_{m}+3 a_{0}\right) .
$$

Goodman works with $\phi$ since in the case of perfect thermal contact it is not possible to determine an initial condition for $a_{0}$. In our case we retain this form since the numerical solution of the resultant differential equation appears more stable with $\phi$ than $a_{0}$. Integrating the heat equation across the solid leads to

$$
\frac{d \phi}{d t}=\frac{3 \kappa_{s}\left(T_{m}-a_{0}\right)}{H_{0}-h_{m}}+a_{0}\left(\frac{d h}{d t}-\frac{d h_{m}}{d t}\right)-T_{m} \frac{d h}{d t},
$$

where $a_{0}$ may be expressed in terms of $\phi$ via Eq. (32). The temperature profile in the water is the same as in stage 2 so the Stefan condition is now

$$
\rho_{s} L_{m} \frac{d h_{m}}{d t}=-\frac{3 k_{s}\left(T_{m}-a_{0}\right)}{H}+\frac{k_{l}\left[q+h_{s l}\left(T_{s}-T_{m}\right)\right]}{k_{l}+h_{s l} h} .
$$

Again the system is closed with the force balance (14). The three equations are solved subject to the initial conditions $h_{m}=h_{m_{2}}, h=h_{2}, \phi=\left(H_{0}-h_{m_{2}}\right)\left(3 \theta_{0}+T_{m}\right) / 4$ at $t=t_{2}$.

\section{RESULTS}

In all the following results we take parameter values from Table I, except when we study the effect of increasing the heat transfer coefficient.

In Fig. 3 we show a comparison of the temperature profiles at the end of stage 1. The exact solution, given by Eq. (21), and the cubic approximation, Eq. (23), are shown as solid and dashed lines, respectively. As mentioned above, the form of the HBI profile in Eq. (23) was taken as a cubic based on the work of Ref. 20. However, there are numerous other possible approximating functions, perhaps the two most standard are the quadratic of Goodman, see Ref. 27, for example, and the exponential approximation of Mosally et al. ${ }^{30}$ In fact, even in the simple case of the quadratic there are seven different formulations. ${ }^{29}$ In Fig. 3 we also show solutions obtained using an exponential and the best quadratic. In agreement with the conclusions of Myers et al. ${ }^{20}$ the cubic approximation is clearly the best. This may also be inferred from the predictions of $t_{1}$. The exact melt time is $t_{1} \approx 1.6354 \mathrm{~s}$ and the melt times predicted for the cubic, quadratic, and exponential profiles are $t_{1} \approx 1.6362$ (with a $0.05 \%$ 


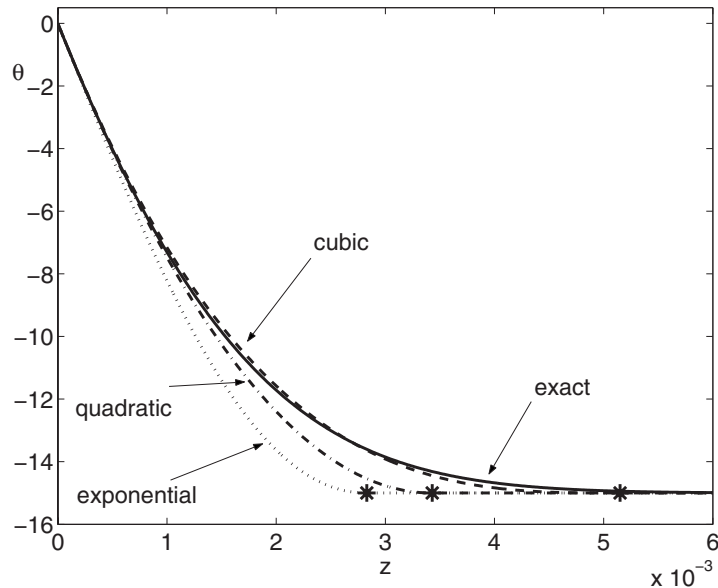

FIG. 3. Temperature profile at the end of stage 1, i.e., $t=t_{1}$. Shown is the exact solution (solid line) compared with the cubic (dashed line), quadratic (dash-dotted line), and exponential (dotted line) heat balance integral method solutions, with $\delta$ marked by “*” for each case.

error), 1.4544 (11\% error), and 1.2635 (23\% error), respectively. Since the cubic is clearly the most accurate approximation in subsequent stages we only consider this profile.

In Figs. 4(a) and 4(b) we present the evolution of the melt height $h_{m}$ and the liquid layer height $h$. Figure 4(a) shows $h_{m}$ obtained by solving the full problem and also the prediction from the quasisteady state solution. The full solution begins at $t_{1} \approx 1.6 \mathrm{~s}$ and shows two stages, for $t_{1} \leq t$ $\leq t_{2}$ (where $t_{2} \approx 79 \mathrm{~s}$ ) it is shown as a dotted line, subsequently for $t_{2} \leq t \leq t_{m}$ (where $t_{m} \approx 1022 \mathrm{~s}$ ), it is a solid line. It is clear that $h_{m}$ is approximately linear with slight deviations near $t=0$ and $t=t_{m}$. The quasisteady solution, Eq. (16), also predicts a linear height increase. The two broken lines

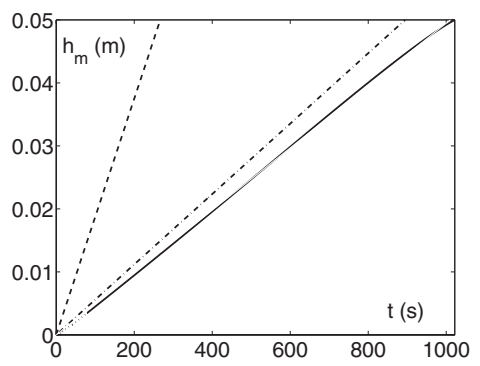

(a)

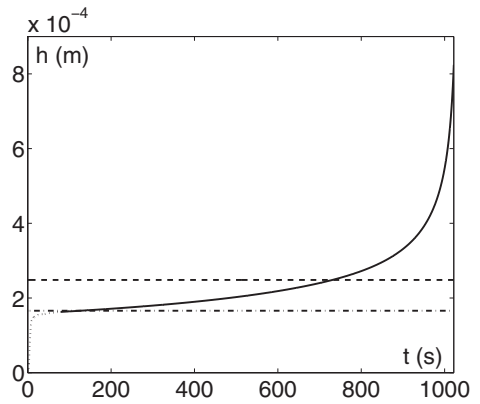

(b)

FIG. 4. (a) $h_{m}(t)$ predicted by current method ( $t<t_{2}$ dotted line, $t>t_{2}$ solid line) and quasisteady solutions, $h_{q \infty}$ (dashed line) and $h_{q}$ (dash-dotted line) for $h_{s l}=855$, (b) melt height predictions; $h(t)\left(t<t_{2}\right.$ dotted line, $t>t_{2}$ solid line), $h_{q_{\infty}}$ (dashed line), and $h_{q}$ (dash-dotted line).

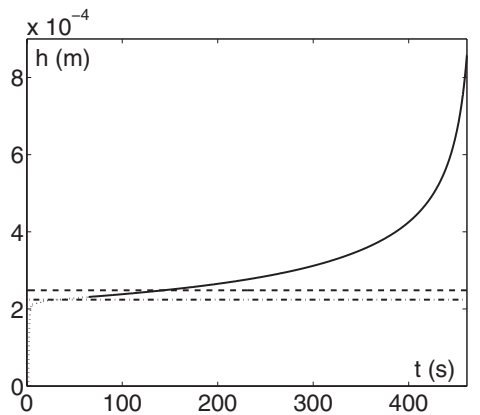

(a)

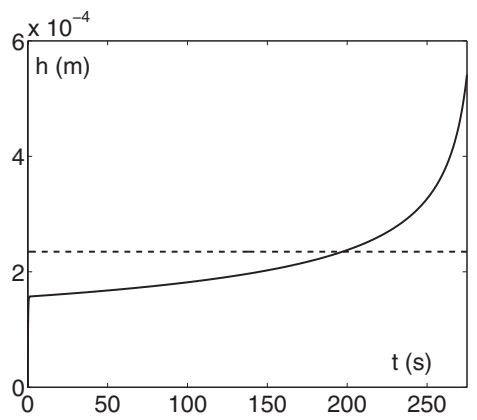

(b)

FIG. 5. (a) $h(t)$ when $h_{s l}=5000$ and quasisteady solutions, $h_{q_{\infty}}$ (dashed line), $h_{q}$ (dash-dotted line) $t_{m} \approx 461 \mathrm{~s}$, (b) $h(t)$ when $h_{s l}=\infty, t_{m} \approx 275 \mathrm{~s}$, and $h_{q \infty}$.

shown take the value of $h_{q}$ [required in Eq. (16)] from either the quartic equation (18) or the perfect thermal contact case (20); these are shown as dash-dotted and dashed lines, respectively. For the perfect thermal contact case the predicted melt time of $t_{m} \approx 267 \mathrm{~s}$ is close to a quarter of the true value. The value of $h_{q}$ from the quartic equation $t_{m} \approx 902 \mathrm{~s}$ leads to an $11 \%$ error in the melt time. The value of $h_{q}$ is determined via the steady state analysis, neglecting the temperature variation in the solid and the changing mass, so at least one of these three factors must be responsible for the discrepancy between the present theory and quasisteady results. In Fig. 4(b) we show the evolution of the melt height $h$. Also shown are the quasisteady state results, with the classical solution $h=h_{q \infty} \approx 2.48 \times 10^{-4} \mathrm{~m}$ and the correction for finite heat transfer, $h_{q} \approx 1.66 \times 10^{-4} \mathrm{~m}$ predicted by Eq. (18). The full solution shows that when the block first starts to melt there is only a very thin insulating liquid layer, consequently $h$ initially increases very rapidly. After approximately $10 \mathrm{~s}$ the increase slows. However, there is never a true quasisteady state. Subsequently there is a slow increase, and finally as the weight of the block becomes very small another rapid increase until the whole block has melted. The slow central increase and final rapid progression have been previously noted by Hirata and Makino ${ }^{18}$ and Lacroix. ${ }^{19}$

In Fig. 5(a) we show the effect of increasing the heat transfer coefficient to $h_{s l}=h_{s s}=5000$ on the evolution of $h(t)$. The form of the curve is similar to the previous example, with an initial rapid increase followed by a slower change and then a final rapid increase. However, melting begins very rapidly, at $t \approx 10^{-7} \mathrm{~s}$, and the initial increase occurs over a much shorter time scale than with the lower value of $h_{s l}$, and so we see the progress toward an initial infinite velocity. 


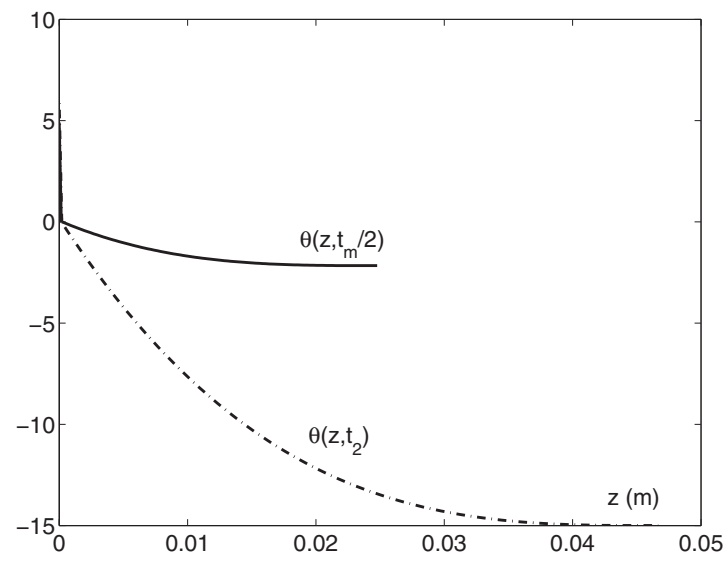

FIG. 6. Temperature in melt and solid at $t=t_{2} \approx 78.5 \mathrm{~s}$ (dash-dotted line) and $t=511 \mathrm{~s}$ (solid line).

Stage 2 ends at $t_{2} \approx 63.8 \mathrm{~s}$. As expected, as $h_{s l}$ increases the present prediction of $h_{q}$ approaches $h_{q \infty}$. In Fig. 5(b) we show the solution for an isothermal block, with an infinite heat transfer coefficient. Equation (30), for $h_{m}$, is singular at $t=0$ (when $h=0$ ), we avoid this by setting $h(0)=10^{-8}$. The singularity carries through to the $h$, Eq. (14), and results in an initial infinite velocity for $h$. Lacroix's ${ }^{19}$ model with heat flow in the solid has perfect thermal contact and also shows an infinite initial velocity. The melting time can be seen to decrease as the heat transfer coefficient increases: When $h_{s l}$ $=855$ we find $t_{m} \approx 1022 \mathrm{~s}$, with $h_{s l}=5000$ we find $t_{m}$ $\approx 461 \mathrm{~s}$ for $h_{s l} \rightarrow \infty, t_{m} \approx 275 \mathrm{~s}$.

In Fig. 6 we show the temperature profiles in the melt layer and solid at the end of stage 2, when $t=t_{2} \approx 79 \mathrm{~s}$ and midway through the melting process, when $t \approx 511 \mathrm{~s}$. At the end of stage 2 , shown as the dash-dotted line, the bottom of the liquid layer is at $5{ }^{\circ} \mathrm{C}$, it then decreases through a very thin layer to the melting temperature. The block has only lost a small amount of its initial mass and the total thickness of melt and block is around $0.0465 \mathrm{~m}$. Midway through the melting process, when the block is around half of its original thickness, shown as the solid line in Fig. 6, the temperature in the liquid layer is similar to that at $t_{2}$ with a maximum temperature of $6{ }^{\circ} \mathrm{C}$, but the solid layer is much hotter, with a minimum temperature of around $2.27{ }^{\circ} \mathrm{C}$.

The reduction in the initially complex system of governing equations was based on the assumption that a number of nondimensional groupings was small. We may now check the accuracy of these assumptions. To simplify the NavierStokes equations we neglected terms of $\mathcal{O}\left(\epsilon^{2} \mathrm{Re}\right)$, and to simplify the heat equation in the liquid we neglected terms of $\mathcal{O}\left(\epsilon^{2} \mathrm{Pe}\right)$. For our example of ice and water $\epsilon^{2} \mathrm{Re} / \epsilon^{2} \mathrm{Pe}$ $\approx 0.135$, and so, provided $\epsilon^{2} \mathrm{Pe}$ is negligible, we can justify neglecting $\epsilon^{2}$ Re. To calculate Pe we require an estimate for the velocity scale. In Fig. 7 we plot the variation in the maximum horizontal fluid velocity with time. The maximum velocity occurs at $(x, z)=[ \pm L, h(t) / 2]$ and is obtained from Eq. (12), $u_{\max }=p_{x} h^{2} /(8 \eta)$, with $p_{x}= \pm 3 F / 2 L^{2}$ [from Eq. (13)]. Two cases are shown, with $h_{s l}=855$ and 5000. Again stage 2 results are shown as a dotted line and stage 3 as a solid line. For the higher heat transfer coefficient we expect
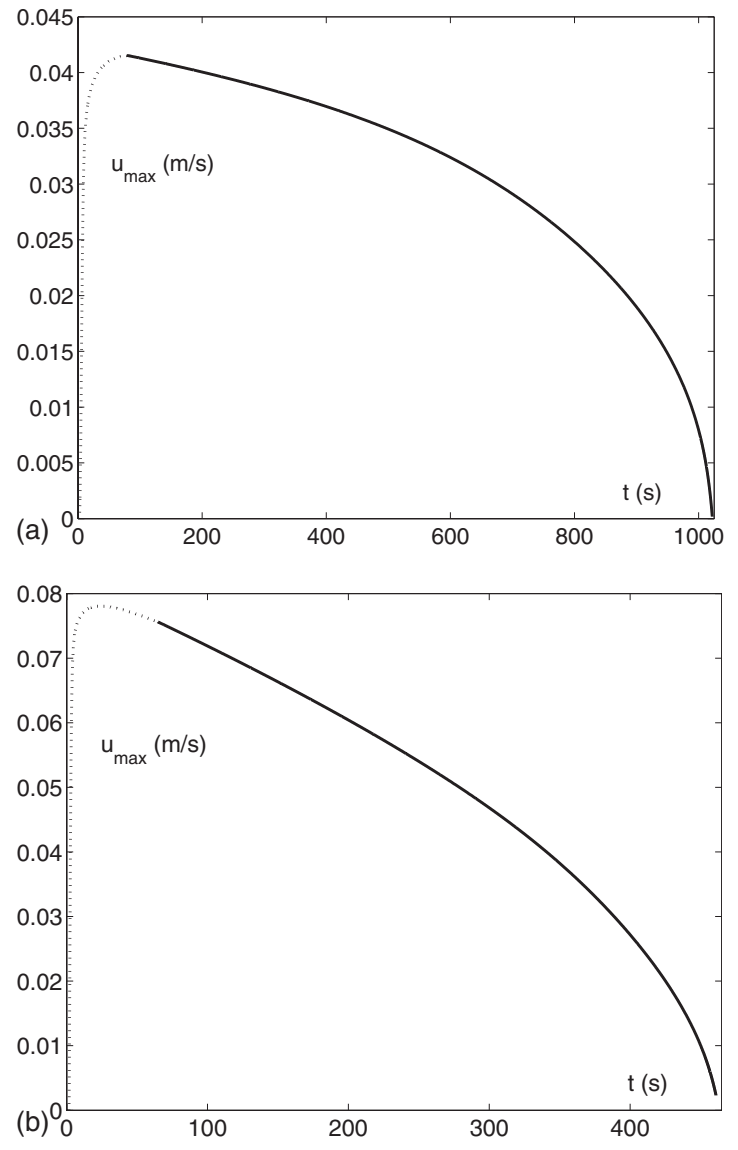

FIG. 7. Maximum horizontal velocity in the melt region for (a) $h_{s l}=855$ and (b) $h_{s l}=5000$.

more rapid melting and consequently a thicker fluid film. This is reflected in the higher fluid velocity, with a maximum close to $8 \mathrm{~cm} / \mathrm{s}$ in the initial phase of melting. The decrease in velocity for larger times is related to the decrease in the mass of solid, thus reducing the driving force. In Fig. 8 we show the variation of

$$
\epsilon^{2} \mathrm{Pe} \sim \frac{h^{2}}{L^{2}} \frac{u_{\max } L}{\kappa_{l}} .
$$

From this we can see that for most of the time $\epsilon^{2} \mathrm{Pe}<0.2$ when $h_{s l}=855$ and $\epsilon^{2} \mathrm{Pe}<0.4$ when $h_{s l}=5000$. The maximum value is occurring in both cases in the very final stage of melting when $h$ approaches a maximum. The decrease at the end reflects the drop in fluid velocity. Since we have chosen the maximum value of $u$ at any time, our value for $\epsilon^{2} \mathrm{Pe}$ is an upper limit and consequently it is reasonable to neglect terms of $\mathcal{O}\left(\epsilon^{2} \mathrm{Pe}\right)$; however, in a perturbation analysis this will usually be the term that should first be reintroduced. This is in keeping with the work of Groulx and Lacroix ${ }^{1}$ and $Y^{1}{ }^{15}$ who both retain the convection term $w T_{z}$.

In the force balance, Eq. (4), we neglected the acceleration term which is $\mathcal{O}\left[\mathcal{H}_{l} /\left(g \tau^{2}\right)\right]$. Since the liquid height is always small this term is only likely to be important when there is a very rapid change. From Figs. 4 and 5 we see that the most rapid variation occurs near $t=0$. If we scale the full force balance [given by Eq. (4) with an acceleration term added], so the acceleration term balances with the fluid pres- 


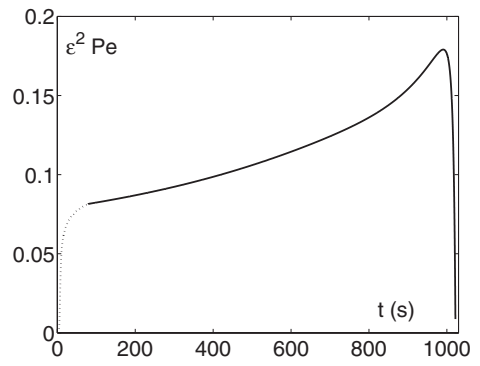

(a)

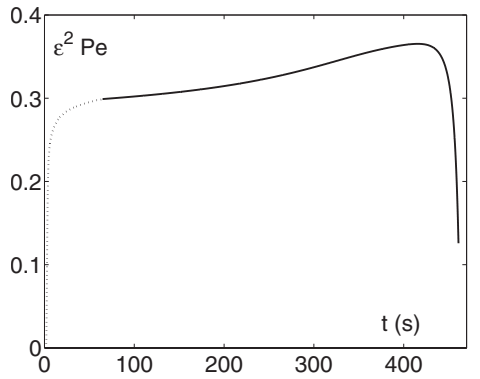

(b)

FIG. 8. Variation of $\epsilon^{2}$ Pe with $t$; (a) $h_{s l}=855$ and (b) $h_{s l}=5000$.

sure, and set $H_{0}-h_{m}=H_{0}$ then we find $\mathcal{H}_{l} \sim\left(2 \eta L \tau / \rho_{l}\right)^{1 / 3}$. If we take the height scale $\mathcal{H}_{l}=h_{q} \sim 2 \times 10^{-4}$ then the acceleration term is only important for time scales $\tau \sim 10^{-5} \mathrm{~s}$. It is therefore reasonable to neglect the acceleration term.

\section{A. Time to complete melting and approximate solutions}

The melting process is complete when $h_{m}=H_{0}$, and we denote this time by $t_{m}$. For the quasisteady model described in Sec. III this is determined by substituting for $h_{m}$ in Eq. (16) to give

$$
t_{m}=\frac{4 \eta L^{2}}{\rho_{l} g h_{q}^{3}},
$$

where $h_{q}$ can be determined by solving the quartic, Eq. (18), or using the approximations given by Eqs. (19) or (20). For the current problem we do not have a simple expression for $h_{m}$, however, from Fig. 4(a) it is clear that $h_{m} \propto t$ throughout the melting period. To identify the constant of proportionality we consider the early part of stage 3 , where $t \approx t_{2}$. From Fig. 4(b) we see that $h \sim h_{q} \ll H$, and at the start of stage $3, H$ $\approx H_{0}$ and $a_{0}=\theta_{0}$ consequently, from Eq. (34) we find

$$
\frac{d h_{m}}{d t} \approx \frac{1}{\rho_{s} L_{m}}\left\{\frac{3 k_{s}\left(\theta_{0}-T_{m}\right)}{H_{0}}+\frac{k_{l}\left[q+h_{s l}\left(T_{s}-T_{m}\right)\right]}{k_{l}+h_{s l} h_{q}}\right\}=\alpha .
$$

Since $t_{1} \ll t_{m}$ we may impose the initial condition $h_{m}(0)=0$ and so integrate this equation to give $h_{m} \approx \alpha t$. The time to melting predicted by this method is therefore

$$
t_{m}=\frac{H_{0}}{\alpha},
$$

where $\alpha$ is defined in Eq. (37). When $h_{s l}=855$ the numerical solution predicts $t_{m} \approx 1022 \mathrm{~s}$, using the quartic solution for

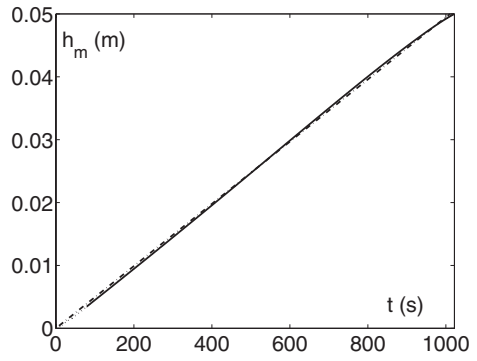

(a)

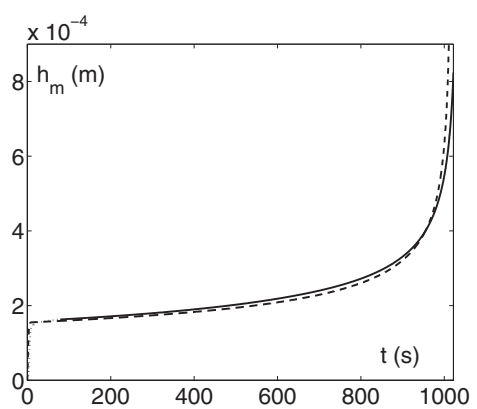

(b)

FIG. 9. Comparison of solutions for (a) $h_{m}(t)$ and (b) $h(t)$. The solid lines represent the exact solution and the dashed lines are the linear approximation.

$h_{q}$ to determine $\alpha$ we find $t_{m} \approx 1010 \mathrm{~s}$ (a $1.2 \%$ error). The quasisteady approximation gives $t_{m} \approx 902$ (an $11 \%$ error).

Equation (37) allows us to calculate the relative magnitude of the conduction terms through the solid and liquid. Using the values in Table I we find that the solid conduction term is a factor of 10 less than the liquid conduction term. This indicates that the subcooling $\theta_{0}$ has a relatively small effect on the process. This could also be inferred from Fig. 6, where it is clear that the temperature gradient in the liquid is much greater than in the solid. Simulations carried out with changing the initial temperature $\theta_{0}$ showed that the melting time shows a slow linear increase with decreasing $\theta_{0}$, in the range of $[-50,0]^{\circ} \mathrm{C}$ (where $t_{m}=1150 \mathrm{~s}$ for $\theta_{0}=-50$ ). Note the fact that the process is driven by the temperature gradient in the liquid also explains why, although the solid temperature is calculated incorrectly in Ref. 19, our results show similar features. Neglect of the mass variation, so $H=H_{0}$ for all time, leads to $t_{m} \approx 940 \mathrm{~s}$. Since both the temperature and mass variation in the solid make relatively small changes to the melting time, we may deduce that the large difference between the present work and previous results may be primarily attributed to the use of a cooling condition, rather than perfect thermal contact.

Finally, given that we have a simple expression for $h_{m}$ that holds (approximately) for all $t>t_{1}$, we are able to substitute this into Eq. (14) to find a single first order differential equation for $h(t)$,

$$
\frac{d h}{d t}=\frac{\rho_{s}}{\rho_{l}} \alpha-\frac{\left(H_{0}-\alpha t\right) \rho_{l} g}{4 \eta L^{2}} h^{3}, \quad h(0)=0 .
$$

The problem of contact melting can therefore be reduced to solving this equation. In Fig. 9(a) we compare the full solution for $h_{m}$ with the linear approximation for $h_{s l}=855$. In Fig. 
9(b) we compare the full solution for $h$ with the numerical solution of Eq. (39). Obviously both comparisons indicate that the simple solution provides an excellent approximation to the solution of the full system.

\section{B. Experimental validation}

Moallemi et al. ${ }^{3}$ carried out experiments on contact melting of $n$-octadecane (the appropriate parameter values are given in Table I). Their theoretical model invokes assumptions (2), (4), and (6). Instead of assumption (1) they used the subcooled solid method where $L_{m} \rightarrow L_{m}+c_{s}\left(T_{m}\right.$ $-\theta_{0}$ ), and instead of assumption (3) they permit vertical convection and so impose a quadratic form for the liquid temperature. In dimensional form their expression for the height of the block $H(t)$ is

$$
\begin{aligned}
H(t)= & L\left[\left(\frac{H_{0}}{L}\right)^{3 / 4}\right. \\
& \left.-\frac{3 \kappa_{l} t}{4 L^{2}}\left\{\frac{g L^{3}}{\kappa_{l}^{2}}\left[\frac{c_{l}\left(T_{s}-T_{m}\right)}{L_{m}+c_{s}\left(T_{m}-\theta_{0}\right)}\right]^{3} \frac{k_{l}}{4 \rho^{2} \eta_{l} c_{l}}\right\}^{1 / 4}\right]^{4 / 3},
\end{aligned}
$$

where $\rho=\rho_{s} / \rho_{l}$. In their analysis they do not specify which density is used in their Stefan condition, however, following through their working shows it to be $\rho_{l}$ (the correct term is $\left.\rho_{s}\right)$. Their expression for the melting velocity [their Eq. (20)] has a $\rho$ in the numerator; in fact, it should be $\rho^{2}$ in the denominator. Similarly their expression for the height [their Eq. (22)] should have $\rho \rightarrow \rho^{-2}$. This change improves the correspondence of their theory with the experimental results. In Eq. (40) we present the corrected version.

Our analysis so far has shown that imposing an infinite heat transfer coefficient gives a too high melting rate. The Stefan condition shows that the melting rate depends on the difference between the temperature gradients in the solid and liquid. Neglecting the gradient in the solid therefore also acts to increase the melting rate. Consequently we would expect the predicted melting rate of Ref. 3 to be greater than the true rate. This is indeed the case, the corrected formula (40) shows a time to complete melting around $15 \%$ faster than experiment (the old formula is around 20\% faster).

One source of error common to both their analysis and the current work is the fact that the analysis is two dimensional, yet the experiments involve a three-dimensional block. To minimize this error they choose a wide block with a base cross section typically $38 \times 230 \mathrm{~mm}$, i.e., an aspect ratio of 0.165 (which is relatively high compared to approximations made in the modeling). Fluid leaking out of the edges that are neglected by the two-dimensional theory will result in a thinner film and consequently a higher melting rate. So a correct two-dimensional theory should, in fact, predict a slower melting rate than is seen in practice (rather than the higher melting rate of the theory in Ref. 3). In Fig. 10 we compare results from the current theory with the experimental data of Ref. 3, Fig. 3. The (corrected) analytical result of Ref. 3 is also shown. The experimental data are marked with asterisks; the present theory with $h_{s l}$ $=7000 \mathrm{~W} / \mathrm{m}^{2}$ is shown as a solid line, and that of Ref. 3 as

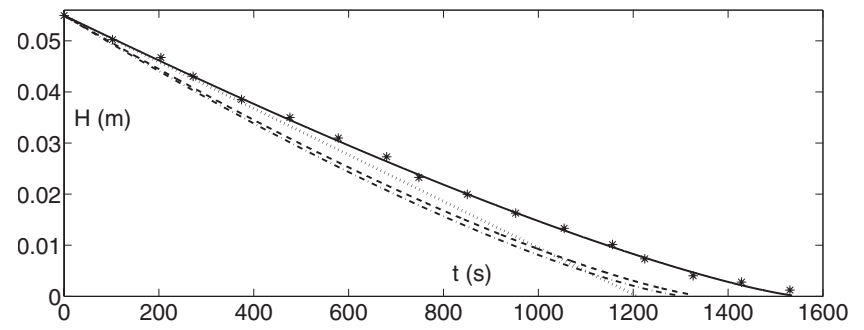

FIG. 10. Comparison of height $H(t)$ with experiment (asterisks) for the current theory with (a) $h_{s l}=7000 \mathrm{~W} / \mathrm{m}^{2}$ (solid line), (b) $h_{s l}=2 \times 10^{6}$ (dashed line), (c) Eq. (40) (dash-dotted line), and (d) quasisteady solution of Eq. (16) (dotted line).

a dash-dotted line. An infinite heat transfer coefficient in the present theory gives the third curve, shown as a dashed line (in fact, we take $h_{s l}=2 \times 10^{6}$; all solutions for $h_{s l}>5 \times 10^{4}$ are identical). The final curve, shown as a dotted line, is the value of $H_{0}-h_{m}$, where $h_{m}$ is the quasisteady solution of Eq. (16). As expected, the quasisteady solution agrees well with the full solution for small times. However, since the mass remains at its initial value the liquid film is squeezed out faster than in reality and so the quasisteady result leads to a melting rate greater than the true value, and the difference increases with time (as the block mass decreases). Our quasisteady result starts off as a better approximation than the infinite heat transfer models which allow for decreasing mass, but in the final stages of melting it ends up as the worst of the models shown. The standard quasisteady model with infinite heat transfer gives even worse correspondence. Note that with $h_{s l}=7000$ we can obtain almost exact agreement with the experiment. In fact, this correspondence is better than we expect; the theory should predict a lower melting rate since it neglects the three-dimensional leakage. The melting rate can be lowered by reducing the value of $h_{s l}$. The infinite heat transfer coefficient result is close to that predicted by Ref. 3 but has a slightly lower melting rate. The difference in results can be attributed to our inclusion of heat flow in the solid. With this high value of $h_{s l}$ the main difference between theories is the heat flow in the solid so this highlights the fact that for this problem, the key factor in predicting the melting is the choice of heat transfer coefficient; the heat flow in the solid plays a relatively small role.

\section{EXTENSION TO THREE DIMENSIONS WITH SLIDING}

Many recent studies have dealt with contact melting in three dimensions, see Refs. 13 and 15, for example. Having established the basic formulation for the two-dimensional problem we now briefly outline the extension to three dimensions; in addition we describe the inclusion of sliding, which is also discussed in Refs. 13 and 15.

For the three-dimensional problem, provided the heat flow is dominated by conduction in the $z$-direction, the only differences in the formulation are to the flow equations and the force balance,

$$
\eta \frac{\partial^{2} u}{\partial z^{2}}=\frac{\partial p}{\partial x}, \quad \eta \frac{\partial^{2} v}{\partial y^{2}}=\frac{\partial p}{\partial y}, \quad \frac{\partial p}{\partial z}=0
$$




$$
\begin{aligned}
& \frac{\partial u}{\partial x}+\frac{\partial v}{\partial y}+\frac{\partial w}{\partial z}=0, \\
& 4 \rho_{s} L W\left[H_{0}-h_{m}(t)\right] g=\int_{-L}^{L} \int_{-W}^{W} p d y d x,
\end{aligned}
$$

where the width of the block is $2 W$. If we allow the top surface to move in the $x$-direction with velocity $U$ then

$$
u=\frac{p_{x}}{2 \eta} z(z-h)+U \frac{z}{h}, \quad v=\frac{p_{y}}{2 \eta} z(z-h) .
$$

Integrating the incompressibility condition from $z=0$ to $h$ we find

$w(h)-w(0)=\frac{\partial h}{\partial t}-\frac{\rho_{s}}{\rho_{l}} \frac{\partial h_{m}}{\partial t}=-\frac{\partial}{\partial x} \int_{0}^{h} u d z-\frac{\partial}{\partial y} \int_{0}^{h} v d z$,

$$
=\frac{h^{3}}{12 \eta} \nabla^{2} p-\frac{U h}{2} .
$$

So we see that the main difference between the two- and three-dimensional models: The pressure satisfies Poisson's equation,

$$
\nabla^{2} p=\frac{12 \eta}{h^{3}}\left(\frac{\partial h}{\partial t}-\frac{\rho_{s}}{\rho_{l}} \frac{\partial h_{m}}{\partial t}+\frac{U h}{2}\right)=f(t),
$$

subject to $p( \pm L, y)=p(x, \pm W)=0$. To determine the pressure we employ an eigenfunction expansion of the form

$$
p=\sum_{n=1}^{\infty} b_{n}(s) \sin \omega_{n} r
$$

where for convenience we set $r=(x+L) / 2, s=(y+W) / 2$ and the eigenvalue $\omega_{n}=n \pi / L$. Substituting this back into Eq. (46) gives

$$
\sum_{n=1}^{\infty}\left(\frac{d^{2} b_{n}}{d s^{2}}-\omega_{n}^{2} b_{n}\right) \sin \omega_{n} s=4 f(t) .
$$

By orthogonality

$$
\frac{d^{2} b_{n}}{d s^{2}}-\omega_{n}^{2} b_{n}=\frac{8 f}{L} \int_{0}^{L} \sin \omega_{n} s d s=q_{n}(t),
$$

where $q_{n}(t)=8 f(t)\left[1-(-1)^{n}\right] /(n \pi)$. Applying the conditions $b_{n}(0)=b_{n}(W)=0$, we determine the eigenfunctions

$$
b_{n}(s)=\frac{q_{n}}{\omega_{n}^{2}}\left[\cosh \omega_{n} s-1+\frac{1-\cosh \omega_{n} W}{\sinh \omega_{n} W} \sinh \omega_{n} s\right] .
$$

The load carried by the fluid is then

$$
\begin{aligned}
F & =\int_{0}^{W} \int_{0}^{L} p(r, s) d r d s \\
& =\sum_{n=1}^{\infty} \frac{q_{n}}{\omega_{n}^{3}}\left[\frac{\sinh \omega_{n} W}{\omega_{n}}-W-\frac{\left(1-\cosh \omega_{n} W\right)^{2}}{\omega_{n} \sinh \omega_{n} W}\right]\left[1-(-1)^{n}\right] .
\end{aligned}
$$

The analysis now proceeds in a similar manner to the twodimensional formulation with the only difference coming through the expression for the force. We replace the force balance, Eq. (14), with

$$
\begin{aligned}
4 \rho_{s} L W\left[H_{0}-h_{m}(t)\right] g= & 8 f(t) \sum_{n=1}^{\infty} \frac{\left[1-(-1)^{n}\right]^{2}}{n \pi \omega_{n}^{3}} \\
& \times\left[\frac{\sinh \omega_{n} W}{\omega_{n}}-W\right. \\
& \left.-\frac{\left(1-\cosh \omega_{n} W\right)^{2}}{\omega_{n} \sinh \omega_{n} W}\right],
\end{aligned}
$$

where $f(t)$ is given by Eq. (46).

\section{CONCLUSION}

A model has been developed to describe unsteady contact melting of a nonisothermal solid from the initial heating up phase to complete melting. In our examples we mainly used data for ice and water since these are the easiest to obtain, however, the governing equations should be valid for other melting systems provided the small parameters remain negligible.

The key differences between our work and most previous models include the following:

(1) The modeling of the temperature profile in the solid.

(2) The inclusion of a cooling condition at the interface with the substrate.

(3) Modeling the varying mass of the solid.

(4) The neglect of the quasisteady assumption for the liquid height.

With these modifications included, the present theory showed almost exact agreement with experimental data.

Modeling the temperature in the solid allowed us to include the solid temperature gradient in the Stefan condition. This term acted to slow down the melting. The time to complete melting slowly increased linearly with a decrease in subcooling, $\theta_{0}-T_{m}$. Since the weight of the solid provides the driving force for the squeeze flow the decreasing mass also leads to an increase in the melt time. Further, the mass variation prevented the quasisteady state from occurring, instead the liquid height has an initial rapid increase, followed by a period of slow increase and a final rapid increase as the block mass approaches zero. The quasisteady approximation provides a reasonable approximation to the central period of slow increase. However, it is the cooling condition and the introduction of a heat transfer coefficient that has the greatest 
effect on the melting. Increasing the heat transfer coefficient from 855 to $5000 \mathrm{~W} / \mathrm{m}^{2}$ halved the melting time. Further, with a cooling condition melting does not occur immediately, so we must examine a premelting stage, which then determines the time for melting to start and also the temperature profile in the solid when this occurs. Under the quasisteady assumption the cooling condition leads to a qualitatively different expression for the melt thickness, $h_{q} \sim\left(T_{s}-T_{m}\right)^{1 / 3}$, as opposed to $\left(T_{s}-T_{m}\right)^{1 / 4}$ which results from assuming perfect thermal contact. The initial infinite velocity observed in previous studies did not show up in our simulations and was shown to be due to the perfect thermal contact assumption.

For our main example the combined effects of modeling the temperature in the solid, using a heat transfer coefficient and a decreasing mass, led to a fourfold increase in the melting time from the quasisteady solution with an infinite heat transfer coefficient. The quasisteady solution with a cooling condition led to an $11 \%$ difference from the full solution. When compared with the experimental results for the block thickness the current method showed almost exact agreement with the correct choice of heat transfer coefficient.

Our results showed that for ice the melting rate is approximately constant, so $h_{m} \approx \alpha t$. A similar result is true for the quasisteady approximation but the constant of proportionality is significantly different. This observation allowed us to determine the constant of proportionality from the Stefan condition so the time for melting may be estimated via a simple analytical expression. The evolution of the melt layer thickness reduced to solving a single first order differential equation for the liquid layer height. The results showed excellent agreement with our full solution for ice and water, and the governing equation is clearly a much simpler prospect to solve than the three coupled differential equations that result from our full analysis.

${ }^{1}$ D. Groulx and M. Lacroix, "Study of close contact melting of ice from a sliding heated flat plate," Int. J. Heat Mass Transfer 49, 4407 (2006).

${ }^{2}$ H. Kumano, A. Saito, S. Okawa, and Y. Yamada, "Study of direct contact melting with hydrocarbon mixtures as the PCM," Int. J. Heat Mass Transfer 48, 3212 (2005).

${ }^{3}$ M. K. Moallemi, B. W. Webb, and R. Viskanta, "An experimental and analytical study of close-contact melting," ASME Trans. J. Heat Transfer 108, 894 (1986).

${ }^{4}$ A. G. Herrmann, Radioaktive Abfälle: Probleme und Verantwortung (Springer, Berlin, 1983).

${ }^{5}$ F. Jackson, "Moving heat sources with change of phase," ASME Trans. J. Heat Transfer 87, 329 (1965).

${ }^{6}$ B. D. Marsh, "On the cooling of ascending andesitic magma," Philos. Trans. R. Soc. London, Ser. A 288, 611 (1978).

${ }^{7}$ M. Bareiss and H. Beer, "An analytical process during melting of an unfixed solid phase change material inside a horizontal tube," Int. J. Heat Mass Transfer 27, 739 (1984).

${ }^{8}$ H. Kumano, A. Saito, S. Okawa, and Y. Yamada, "Direct contact melting with asymmetric load," Int. J. Heat Mass Transfer 48, 3221 (2005).

${ }^{9}$ D. Nicholas and Y. Bayazitoglu, "Thermal storage of phase-change material in a horizontal cylinder," in Alternative Energy Sources, Vol. 1(3), Miami International Conference on Alternative Energy Sources, 15 December 1983, p. 351.

${ }^{10}$ A. Bejan, "Contact melting heat transfer and lubrication," Adv. Heat Transfer 24, 1 (1994).

${ }^{11}$ A. L. Biance, C. Clanet, and D. G. Quere, "Leidenfrost drops," Phys. Fluids 15, 1632 (2003).

${ }^{12}$ G. Bleiker and E. H. Specht, "Film evaporation of drops of different shapes above a horizontal plate," Int. J. Therm. Sci. 46, 835 (2007).

${ }^{13} \mathrm{~A}$. Bejan, "The fundamentals of sliding contact melting and friction," ASME Trans. J. Heat Transfer 111, 13 (1989).

${ }^{14}$ P. A. Litsek and A. Bejan, "Sliding contact melting: The effect of heat transfer in the solid parts," ASME Trans. J. Heat Transfer 112, 809 (1990).

${ }^{15} \mathrm{H}$. Yoo, "Analytical solutions to the unsteady close-contact melting," Int. J. Heat Fluid Flow 43, 1457 (2000).

${ }^{16} \mathrm{H}$. Yoo, H. Hong, and C. J. Kim, "Effects of transverse convection and solid-liquid density difference on the steady close-contact melting," Int. J. Heat Fluid Flow 19, 368 (1998).

${ }^{17}$ T. R. Goodman, "The heat-balance integral and its application to problems involving a change of phase," Trans. ASME 80, 335 (1958).

${ }^{18}$ T. Hirata and Y. Makino, "Analysis of close-contact melting for octadecane and ice inside isothermally heated horizontal rectangular capsule," Int. J. Heat Mass Transfer 34, 3097 (1991).

${ }^{19}$ M. Lacroix, "Contact melting of a phase change material inside a heated paralleledic capsule," Energy Convers. Manage. 42, 35 (2001).

${ }^{20}$ T. G. Myers, S. L. Mitchell, G. Muchatibaya, and M. Y. Myers, "A cubic heat balance integral method for one dimensional melting of a finite thickness layer," Int. J. Heat Mass Transfer 50, 5305 (2007).

${ }^{21}$ T. G. Myers, J. P. F. Charpin, and S. J. Chapman, "The flow and solidification of a thin fluid film on an arbitrary three-dimensional surface," Phys. Fluids 14, 2788 (2002).

${ }^{22}$ T. G. Myers, J. P. F. Charpin, and C. P. Thompson, "Slowly accreting ice due to super cooled water impacting on a cold surface," Phys. Fluids 14, 240 (2002).

${ }^{23}$ H. S. Carslaw and J. C. Jaeger, Conduction of Heat in Solids (Oxford University Press, London, 1947).

${ }^{24}$ S. L. Mitchell and T. G. Myers, "Approximate solution methods for one dimensional solidification from an incoming fluid," Appl. Math. Comput. 202, 311 (2008)

${ }^{25}$ T. G. Myers, "Extension to the Messinger model for aircraft icing," AIAA J. 39, 211 (2001).

${ }^{26}$ T. G. Myers and J. P. F. Charpin, "A mathematical model for atmospheric ice accretion and water flow on a cold surface," Int. J. Heat Fluid Flow 47, 5483 (2004)

${ }^{27}$ T. R. Goodman. "Application of integral methods to transient nonlinear heat transfer," Adv. Heat Transfer 1, 51 (1964).

${ }^{28} \mathrm{~S}$. L. Mitchell and T. G. Myers, "Heat balance integral method for onedimensional ablation," J. Thermophys. Heat Transfer 22, 508 (2008).

${ }^{29}$ A. S. Wood, "A new look at the heat balance integral method," Appl. Math. Model. 25, 815 (2001).

${ }^{30}$ F. Mosally, A. S. Wood, and A. Al-Fhaid, "An exponential heat balance integral method," Appl. Math. Comput. 130, 87 (2002). 